\author{
УДК 621.791 .75
}

Микалаускас Г. ${ }^{1}$, Вишняков Н. ${ }^{1}$, Квасницкий В. В. ${ }^{2}$, Стреленко Н. М. ${ }^{2}$

${ }^{1}$ Вильнюсский технический университет имени Гедиминаса. Литва, г. Вильнюс

${ }^{2}$ Национальный технический университет Украины «Киевский политехнический институт имени Игоря Сикорского». Украина, г. Киев

\title{
МИКРОКОМПОЗИТНЫЕ Cu-Nb ПРОВОДНИКИ И ВОЗМОЖНОСТИ ИХ НЕРАЗЬЕМНОГО СОЕДИНЕНИЯ
}

\begin{abstract}
Установки сильных магнитных полей широко применяются в различных областях научных исследований и промышленности. Важнейшим компонентом всех магнитных систем является индуктор (соленоид). Для создания сильного магнитного поля уже недостаточно одного только усовершенствования конструкции соленоидов. Традиционные электротехнические материалы и проводники неспособны выдерживать колоссальные механические и температурные нагрузки, поэтому на смену им приходят совериенно новые перспективные материалы, к которым относятся и микрокомпозитные $\mathrm{Cu}-\mathrm{Nb}$ проводники. Эти микрокомпозитные материаль отличаются уникальной структурой, высокими механическими характеристиками и хорошей электропроводностью. Одной из важнейших нерешенных проблем остается создание надежных неразьемных соединений и поиск новых технологий их изготовления, поскольку большинство таких магнитных установок после начала их әксплуатации должны оставаться неразъемными. В настоящее время для соединения проводников обычно применяют только разъемные резъбовые или паяные соединения, не отличающиеся на практике высокой надежностью. В данной работе представлен обзор: особенностей структуры и характеристик Cu-Nb проводников, применяемых в установках для сильных магнитных полей; спеиифики производства данных композитных проводников; технологических проблем при их соединении сваркой; результов экспериментальных исследований свойств сварных соединенений, которые были выполнены, методом термитной сварки. [dx.doi.org/10.29010/084.6]
\end{abstract}

Ключевые слова: Cи-Nb проводники; электроконтакные соединения; микрокомпозитные материалы; сварное соединение.

\section{1. Введение}

В настоящее время магнитные поля широко применяются в различных отраслях науки, промышленности и медицины. Это быстро развивающаяся область науки и технологий, в которой используются импульсные и постоянные магнитные поля, где магнитная индукция достигает 100 Тл [1]. Сильные магнитные поля стали одним из чрезвычайно важных инструментов в науке, поскольку они создают уникальные эффекты: левитацию, структурные изменения и фазовые превращения материалов, магнитную сепарацию, магнитную анизотропию, суперпроводимость и т.д.. Все большее число представителей различных направлений науки включается в эту сферу исследований, а научно-исследовательские лаборатории и научные центры разных стран, обладающие передовым опытом в области этих технологий, кооперируются для реализации уникальных совместных международных научных проектов [2]. Наиболее известные и перспективные проекты, в которых используются магнитные поля: экспериментальный термоядерный реактор (ITER) и другие токамаки, большой адронный коллайдер в лаборатории CERN, компактный реактор ARC нового поколения. Сравнивая постоянные, импульсные или сверхпроводящие магниты, следует отметить, что импульсные технологии для генерирования сильных магнитных полей являются весьма привлекательными, поскольку не требуют таких крупных инвестиций, как две другие вышеназванные уникальные системы [1]. Для того чтобы сгенерировать магнитное поле более чем в 2 Тл уже необходимы сверпроводящиие соленоиды или другие сложные гибридные системы. Самое сильное постоянное магнитное поле, которое в 2003 г. удалось создать при помощи гибридного магнита, достигло 45 Тл, в 2011 г. при помощи сверхпроводящего магнита удалось создать поле в 35,4 Тл, а в 2010 г. за счет применения резистивного магнита - в 36,2 Тл. Магнитные поля плотностью более 45 Тл могут генерироваться только в виде коротких импульсов. С помощью 
импульсных магнитов достигнута рекордная плотность поля 100 Тл при длительности импульса $10 \mu \mathrm{s}$ без разрушения самого магнита. Этот рекорд был установлен в Национальной лаборатории ЛосАламоса в 2012 г. при тестировании импульсного магнита, в котором были использованы микрокомпозитные $\mathrm{Cu}-\mathrm{Nb}$ проводники [4].

\section{2. Структура, характеристики и области применения микрокомпозитных $\mathrm{Cu}-\mathrm{Nb}$ проводников}

Выбор проводника для магнита определенной конструкции зависит от характеристик магнитного поля, которое планируется сгенерировать. Особенно важны две характеристики проводников, применяемых в индукторах магнитных полей - это прочность и электропроводность. До 1980 г. для намотки индукторов применялись только медные проводники. Медь - хороший проводник тепла и электрического тока, но этот материал обладает малой механической прочностью, медная обмотка индуктора не выдерживает воздействия сил Лоренца [5]. Серьезный прорыв в этой области был сделан только после появления новых проводников из металлических композитов. Материалы, предназначенные для обмотки соленоидов, генерирующих магнитное поле в 5-100 Тл, должны быть прочными (более $750 \mathrm{MПа)} \mathrm{и} \mathrm{хорошими} \mathrm{проводниками} \mathrm{(электропро-}$ водность - свыше 60-70 \% IACS (International Annealed Copper Standard - стандарная электропроводность чистой меди). Высокая прочность необходима, для того чтобы выдержать действие колоссальных сил Лоренца, а хорошая электропроводность - для минимизации нагревания проводников вследствие протекания сильного электического тока. Эти материалы также должны отличаться такой пластичностью, которая позволит избежать излома и обрывов проводников при формировании обмотки индуктора (Gluchovski et al. 2014). Проводники на основе микрокомпозитов $\mathrm{Cu}-\mathrm{Nb}$ в данный момент являются лучшими среди аналогичных проводников, поскольку отличаются особенно высокой прочностью и электроводностью. Их прочность достигает 1100-1500 МПа (у обыкновенного $\mathrm{Cu}$ проводника - 270 МПа), предел текучести 850 МПа, электропроводность - 67-70 \% IACS. Поэтому микрокомпозиты $\mathrm{Cu}-\mathrm{Nb}$ считаются одними из самых перспективных и подходящих из существующих ныне материалов для создания систем генерации сильных импульсных магнитных полей. Структура микрокомпозитных $\mathrm{Cu}-\mathrm{Nb}$ проводников (рис. 1) состоит из медной матрицы, в которую внедрены очень тонкие ниобиевые волокна.

Такие волокна упрочняют медную матрицу и обеспечивают более высокую прочность проводника, при этом, не препятствуя движению электронов.

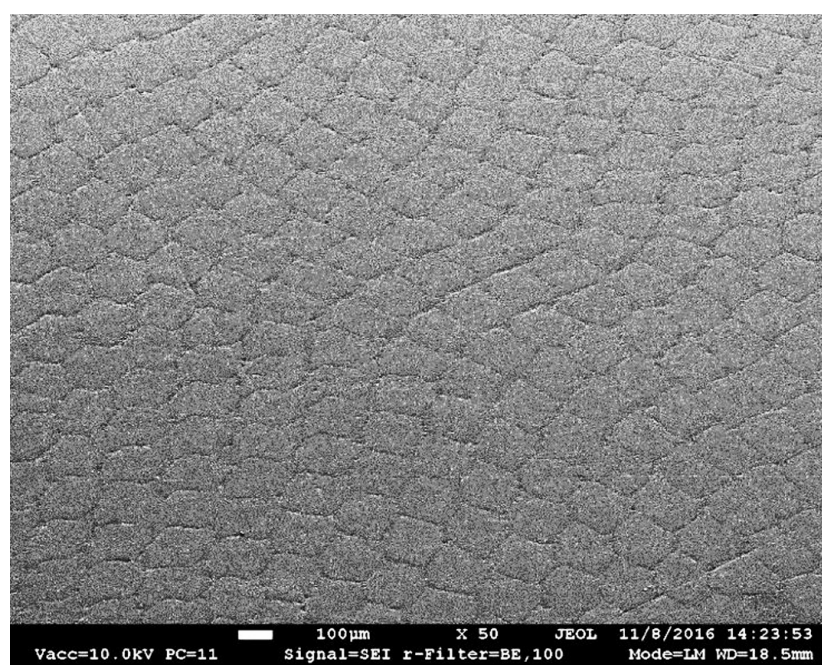

Рис.1. Микроструктура $\mathrm{Cu}-\mathrm{Nb}$ проводника (х50)

\section{3. Особенности производства микрокомпозитных $\mathrm{Cu}-\mathrm{Nb}$ проводников}

Технология производства микрокомпозитных $\mathrm{Cu}-\mathrm{Nb}$ проводников сложна, требует высокой точности и последовательности. В настоящее время при изготовлении микрокомпозитных $\mathrm{Cu}-\mathrm{Nb}$ проводников применяются две производственные технологии:

1. Сплавление материалов различной механической прочности в микро- или нанокомпозит - метод in situ [8];

2. Скрутка волокон проводников хорошей проводимости с волокнами механически высокопрочного материала в один микро- или нанокомпозитный проводни [7].

В первом случае процесс производства проводников «плавление-деформация» (in situ) напоминает традиционную технологию производства многожильных металлических кабелей. Чистая медь и ниобий сплавляются в определенной пропорции. Из полученного сплава $\mathrm{Cu}-\mathrm{Nb}$ в процессе вакуумной плавки методом экструзии изготовляются 20-50 миллиметровые стержни. Эти заготовки в несколько этапов деформируются до получения проволоки нужного диаметра. В результате такого многократного растяжения проволоки из сплава $\mathrm{Cu}-\mathrm{Nb}$ можно получить жилы очень малого сечения (до $90 \mu \mathrm{m}$ ). Структура $\mathrm{Cu}-\mathrm{Nb}$ композита in situ: $\mathrm{Nb}$ жилы в $\mathrm{Cu}$ металлической матрице. Эти жилы позднее покрываются медной оболочкой [6]. Предел прочности такого композитного кабеля очень велик, может составлять 1 ГПа, а электропроводность 60-70 \% IACS.

Другой способ изготовления композитных проводников «сборка-деформация» напоминает производство биметаллов. Свивание медных трубок и $\mathrm{Nb}$ стержней способом сильной многократной деформации. На первом этапе подготавливаются заготовки медных трубок и $\mathrm{Nb}$ стержней. Для этого 


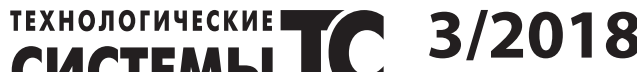

используется сырье технической чистоты, размеры заготовок должны быть очень точные, поэтому трубки и стержни обычно калибруются. На следующем этапе $\mathrm{Nb}$ стержни вставляются в $\mathrm{Cu}$ трубки и повторно вальцуются, заготовки $\mathrm{Cu}$ и $\mathrm{Nb}$ соединяются. На третьем этапе несколько спрессованных $\mathrm{Cu}-\mathrm{Nb}$ композитных заготовок вставляются в медную трубку и снова вальцуются. На четвертом этапе полученные заготовки вновь вставляются в медную трубку, вальцуются и в течение 3 часов выдерживаются при температуре $500{ }^{\circ} \mathrm{C}$. Этот этап нужно повторять до тех пор, пока не получится микрокомпозит нужной структуры [9]. Структура микрокомпозита: $\mathrm{Nb}$ волокна $<15$ нм и медная матрица, расстояние между $\mathrm{Nb}$ волокнами $<60$ нм. На последнем этапе полученная заготовка еще раз вальцуется до необходимого поперечного сечения (от 0,01 до $125 \mathrm{mм}^{2}$ ) и проводники в течение 1 часа выдерживаются при температуре $500{ }^{\circ} \mathrm{C}$. В $\mathrm{Cu}-\mathrm{Nb}$ проводнике сечением $2 \times 3$ мм насчитывается около 400 млн. Nb жил. Основной недостаток изготовляемых первым способом $\mathrm{Cu}-\mathrm{Nb}$ проводников - трудности при наматывании магнитной катушки или их сгибании из-за повышенной хрупкости такого проводика. Проводники, изготовленные вторым способом, могут подвергаться сгибанию, при этом трудно ломаются, предел их прочности составляет 1,5 ГПа, электропроводность 65-75 \% IACS. Механические характеристики этих проводников планируется улучшить и достичь прочности до 2 ГПа и, соответственно, без ухудшения электропроводности [7].

\section{4. Возможности соединения микрокомпозитных проводников}

Конструктивные решения сверхпроводящих систем с сильными магнитными полями предусматривают большое количество соединений между секциями магнитов или модулями оборудования [12]. Наличие соединений диктуется и ограниченной гибкостью микрокомпозитных проводников, модульной структурой магнитных соленоидов и необходимостью возможного ремонта таких дорогостоящих и сложных конструкций, т.е. разборки нужной части магнитов и замены только сгоревших частей магнита. Соединения секций или выводов магнитных катушек не должны быть самым слабым местом магнитных установок. Аналогичные проблемы возникнут и при применении этих проводников и в других областях, например на высоковольтных линиях, где также возникнет необходимость надежного соединения проводов. Структура композитных проводников во время соединения их сваркой плавлением нарушается или изменяется $[13,14]$. Поэтому большинство традиционных методов сварки плавлением не может быть применено для этих композитных материалов, поскольку при плавлении матрицы происхо- дят необратимые структурные изменения. Решением этой проблемы, гипотетически возможно применяя методы диффузионной сварки или сварки давлением, а также других специализированных методов сварки или родственных процессов. Процесс производства микрокомпозитов аналогичен процессу диффузионной сварки или сварки давлением. $\mathrm{Cu}-\mathrm{Nb}$ композит формируется путем многократной механической обработки давлением. При этом деформируемые поверхности сближаются на расстояние возникновения межатомных связей и свариваются, поэтому такие технологии сварки в принципе могут быть применимы и для соединения проводников.

\section{5. Особенности термитной сварки}

Термитная сварка - метод по сути дела давно известный. Его в основном применяют в области транспорта для сварки рельсов, в электротехнике для сварки электрических кабелей и кабелей заземления. Среди других способов сварки термитный выделяется высокой скоростью сварки, независимостью от источника электроэнергии или газа, простотой оборудования, возможностью выполнять соединения в сложных условиях. В данном случае детали свариваются с помощью термита, т.е. металлом, расплавленным в результате химической экзотермической реакции с интенсивным выделением тепла (температура реакции достигает 2300-2700 ${ }^{\circ} \mathrm{C}$ ).

Для соединения проводников применяется термитная сварка способом промежуточного литья с использованием специальных огнеупорных форм и пиропатронов с термитной смесью. Анализируя информацию, которая представлена в патентах разных стран, можно заметить, что в состав термитной смеси, обычно применяющейся для сварки многожильных и сплошных медных проводов, входит до 80 \% оксида меди и 12 \% медного порошка, $11 \%$ алюминия или его сплава, 18 \% других добавок. Еще одним из компонентов, которые используются в смесях для термитной сварки сплавов меди, может быть Sn. Смесь $\mathrm{Sn}$ и $\mathrm{CuO}$ также относится к термитам. Согласно диаграмме Эллингема в результате реакции можно также редуцировать $\mathrm{Cu}$, однако в результате этой экзотермической реакции выделяется небольшое количество тепла. Для дискового вкладыша обычно используется фосфористая дезоксидированная медь, за счет раскислительных свойств которой улучшается качество соединения. Могут также использоваться и стальные дисковые вкладыши. Небольшое количество примеси Fе не ухудшает свойства термитного сварного соединения. Учитывая, что основу микрокомпозитных $\mathrm{Cu}-\mathrm{Nb}$ проводников составляет медь, аналогичные технические решения и термитные смеси могут применяться и для их соединения. 


\section{6. Методика сварки и исследований свойств сварных соединений}

В процессе исследований использовался $\mathrm{Cu}-\mathrm{Nb}$ (82\%-18\%) проводник сечением $2,4 \times 4,2$ мм, изготовленный методом «сборки-деформации». Механические характеристики этого $\mathrm{Cu}-\mathrm{Nb}$ проводника: $R_{0.2}=830-850$ МПа, $R_{a}=1120$ МПа. Структура микрокомпозита: $\mathrm{Nb}$ волокна $<15$ нм и медная матрица. Рецептура термитной смеси была подобрана, исходя из состава известных термитов, применяемых для сварки сплавов меди, и состава $\mathrm{Cu}-\mathrm{Nb}$ микрокомпозита. В настоящее время в мире наиболее популярны смеси для термитной сварки сплавов меди, в составе которых кроме $\mathrm{CuO}$ или $\mathrm{Cu}_{2} \mathrm{O}$ и $\mathrm{Al}$ используется $\mathrm{CaSi}_{2}$ (обычно более $5 \%$ массы). Чаще всего на практике применяется комбинация $\mathrm{Al}$ и $\mathrm{CaSi}_{2}$ в соотношении $2: 1$. В таком случае в ходе термитной реакции формируется аморфный шлак, имеющий состав минерала анортита $\left(\mathrm{CaAl}_{2} \mathrm{Si}_{2} \mathrm{O}_{8}\right)$. Его плотность - $2700 \mathrm{\kappa г} / \mathrm{m}^{3}$, температура плавления $-1550{ }^{\circ} \mathrm{C}$. Состав $\mathrm{CaO}-\mathrm{Al}_{2} \mathrm{O}_{3}-\mathrm{SiO}_{2}$ в таком шлаке по диаграмме Ренкина выраженый в процентах массы: $\mathrm{CaO}-20,14 \%, \mathrm{SiO}_{2}-43,7 \%, \mathrm{Al}_{2} \mathrm{O}_{3}-$ $36,69 \%$. Образование аморфного шлака очень полезно, поскольку в процессе большинства реакций термитных смесей образуется керамический шлак $\mathrm{Al}_{2} \mathrm{O}_{3}$. Исследования Yi Hu Chun продемонстрировали, что для полного отделения шлака адиабатическая температура реакции термитной смеси (максимальная температура, которая может быть достигнута при горении смеси) должна быть выше $2083^{\circ} \mathrm{C}$. Эта температура также не должна превышать $2573{ }^{\circ} \mathrm{C}$, поскольку в противном случае будет генерироваться слишком много паров меди. Кроме того, температура этой реакции должна быть выше температуры плавления всех присутствующих в смеси металлов, но не должна превышать температуру их кипения. Известно, что температура плавления $\mathrm{Cu}$ составляет $1085^{\circ} \mathrm{C}$, температура

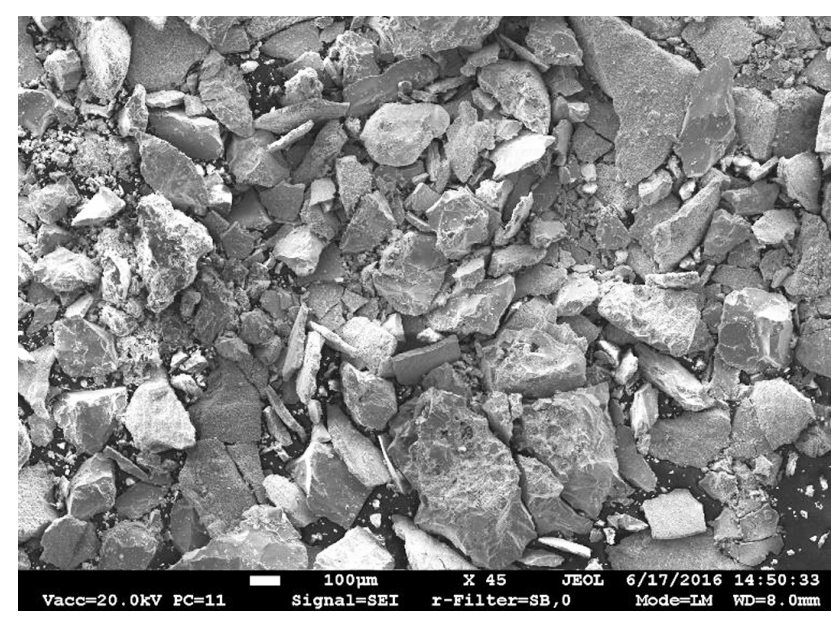

Рис. 3. Вид фракций термитной смеси (х45)

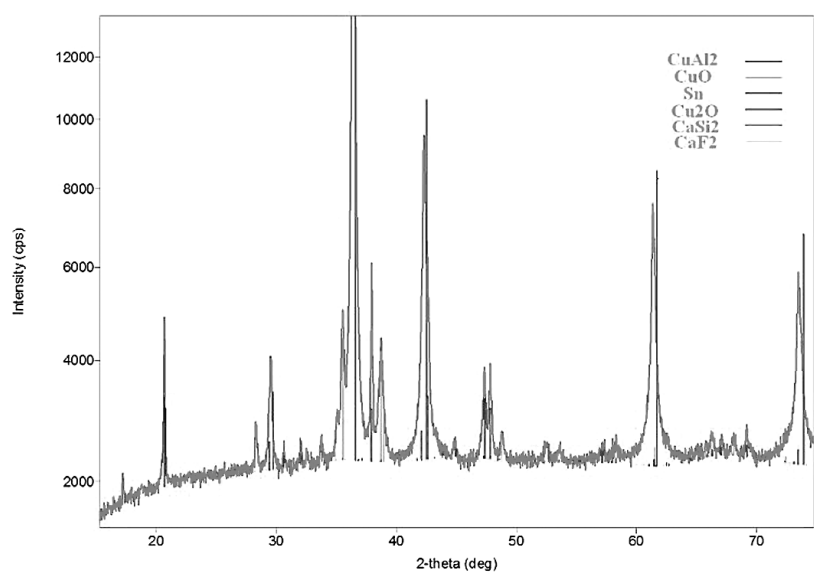

Рис. 2. Результаты XRD-анализа фазового состава термитной смеси

кипения $-2567{ }^{\circ} \mathrm{C}$. Учитывая вышеизложенные условия и составленные уравнения экзотермической реакции, для экспериментов была выбрана термитная смесь, которая должна была бы обеспечить высокую температуру реакции $\left(2449^{\circ} \mathrm{C}-\right.$ $\left.2567{ }^{\circ} \mathrm{C}\right)$. В ходе реакции $\mathrm{Cu}$ образуется до $85 \%$, шлака - не менее 15 \% массы, молярное соотношение между $\mathrm{Al}$ и $\mathrm{CaSi}_{2}$ составляет 2:1. Фазовый состав используемой термитной смеси: $82 \% \mathrm{Cu}_{2} \mathrm{O}$, $8.65 \% \mathrm{CuAl}_{2}, 6.9 \% \mathrm{CaSi}_{2}, 2.2 \% \mathrm{Sn}$ и $0.25 \% \mathrm{CaF}_{2}$. Фазовый состав образцов был установлен путем $\mathrm{XRD}$-анализа при помощи дифрактометра рентгеновского излучения SmantLab (Rigaku) с 9-кВт источником излучения с вращающимся $\mathrm{Cu}$ анодом. Для анализа использована база данных кристаллических соединений PDF-4+ (2013). Результаты $\mathrm{XRD}$-анализа представлены на рис. 2.

Для термитной сварки стыкового соединения $\mathrm{Cu}-$ $\mathrm{Nb}$ проводов (с промежуточным литьем) была изготовлена специальная огнеупорная графитовая форма, общий вид которой представлен на рис. 4. Порция термита, соответствующая объему соединения, заливалась в тигель и поджигалась. После сгора-

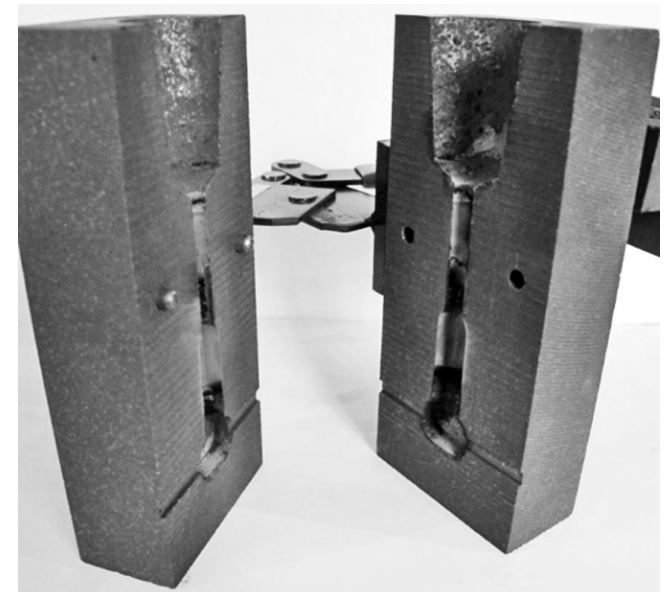

Рис. 4. Общий вид графитовой формы 


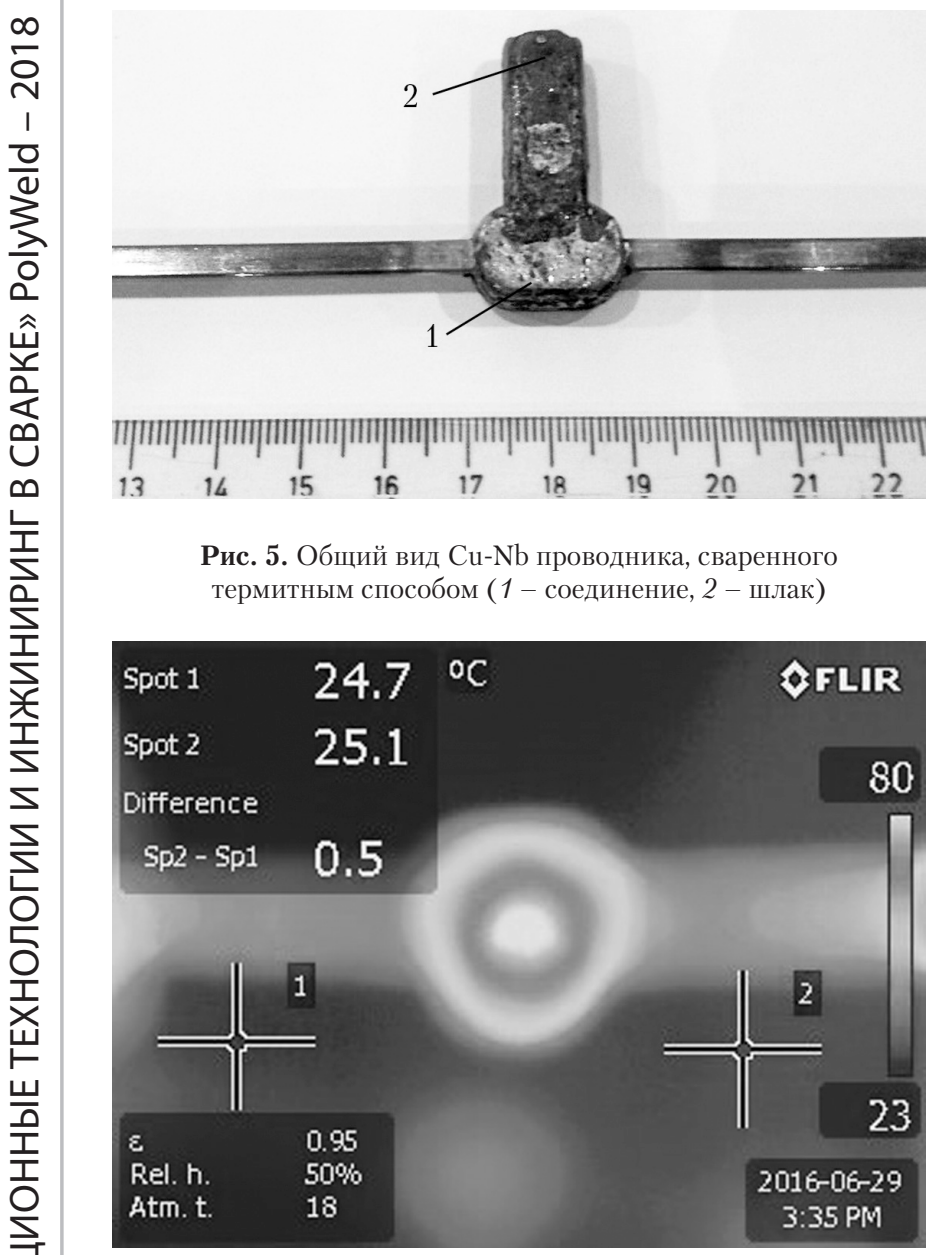

Рис. 6. Распределение температуры в сварном соединении после 2 мин. нагрева

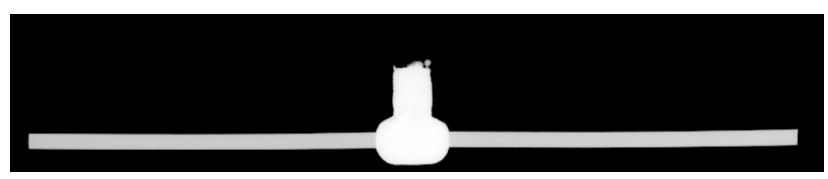

Рис. 7. Рентгеновский снимок термитного сварного соединения

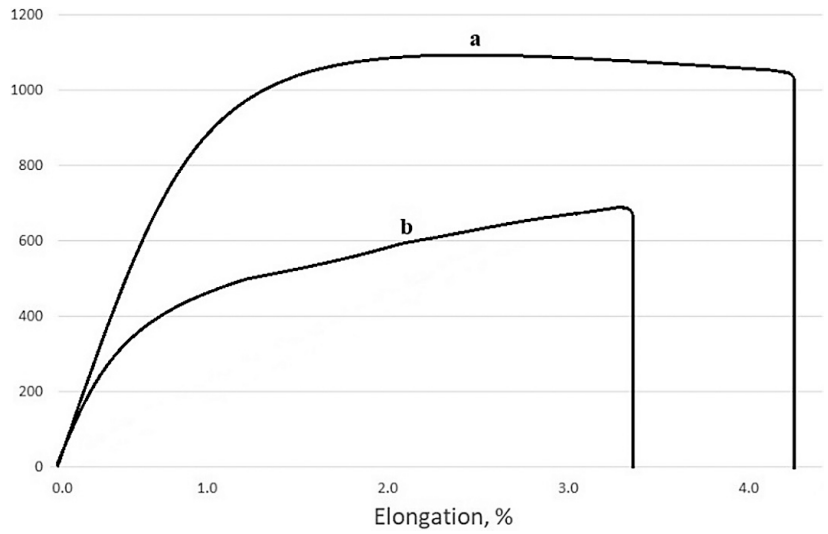

Рис. 8. Испытания на растяжение термитного сварочного соединения:

1 - Cu-Nb проводник; 2 - Cu-Nb проводник, полученый термитной сваркой (место разрыва - основной металл) ния термита тигель наполняется расплавленным металлом. Пока жидкий металл не расплавил дисковый вкладыш, жидкий расплав успел расслоиться в зависимости от плотности компонентов. Нижний слой - жидкий металл, верхний - жидкий шлак, в составе которого преимущественно остаются оксиды.

Для определения качества сварного соединения был выполнен его радиографический контроль с помощью ERESCO 42MF. Механические характеристики были установлены в результате проведенных испытаний на растяжение сварного соединения. Испытания проводились, используя универсальный пресс TIRAtest 2300 с компьютерной измерительной системой, аналого-цифровым преобразователем Spider-8, программным обеспечением catman-Express, динамометром до 100 кН (точность 0,5 \%). Анализ микроструктуры термитного сварочного соединения проводили с помощью метода оптической микроскопии и сканирующей микроскопии (SEM). В процессе исследований применялись сканирующий микроскоп JEOL JSM7600 с анализатором Oxford INCA Energy X-Max20, a также лабораторный оптический микроскоп Nikon MA-200 с цифровой камерой.

\section{7. Результаты исследований}

После подбора композиции термитной смеси, предназначенной для сварки $\mathrm{Cu}-\mathrm{Nb}$ проводника, были проведены сварочные испытания. Общий вид полученного сварного соединения представлен на рисунке 5.

Соотношение металла и шлака, образующихся в процессе термитной сварки, установлено путем взвешивания продуктов реакции. В ходе реакции $\mathrm{Cu}$ получается 76 \%, шлака - 24 \% массы. Сравнение электрических характеристик сварного соединения и проводника было выполнено путем измерения разницы их электрического сопротивления. Разница активного сопротивления проводника и проводника с соединением одинаковой длины (коэффициент дефектности) не достигала 1,2 (соответственно 0,01 Ом и 0,011 Ом). Это не превышает значения, рекомендуемого при термитной сварке. Нагрев сварного соединения под воздействием электрического тока регистрировался с помощью термовизора. Картина, зафиксированная перед началом эксперимента и через 2 минуты нагрева, представлена на рис. 6 . Разница температуры в соединении и проводнике под воздействием электротока не превышала рекомендуемых в нормативных документах значений.

Качество сварного соединения оценивалось с помощью радиографического неразрушающего контроля. Во время контроля внутренние дефекты не были замечены (рис. 7). Результаты испытаний механических характеристик соединения представлены на рис. 8. Прочность соединения составила 701 МПа, 


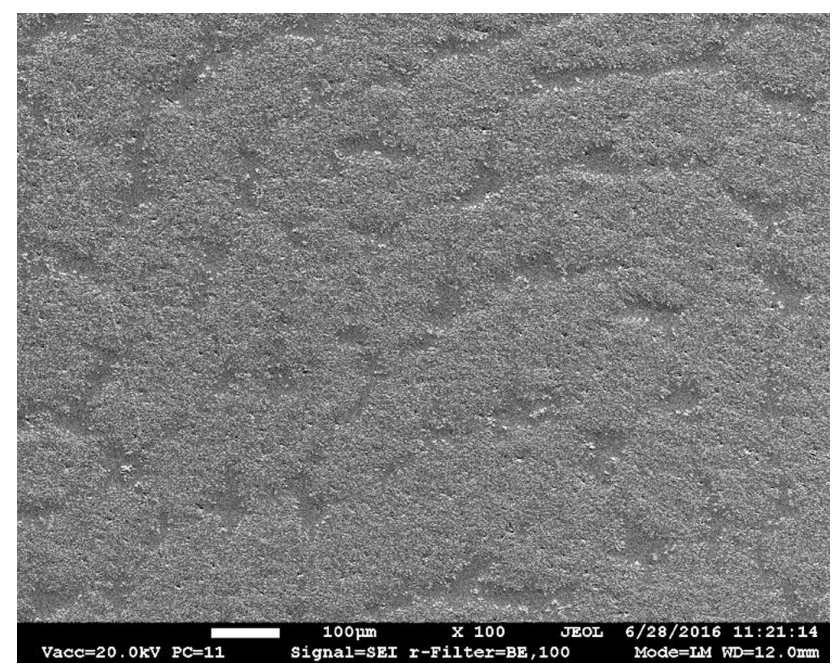

a)

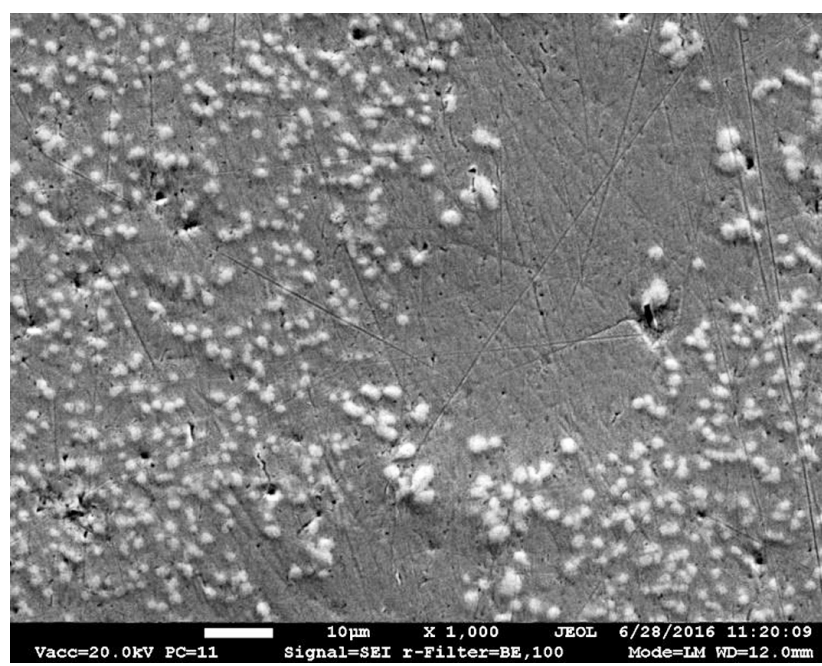

б)

Рис. 9. Микроструктура термитного сварочного соединения ( $a-\mathrm{x} 100, \sigma-\mathrm{x} 1000)$

относительное удлинение - 3,2 \%. Достигнутая прочность соединения составляет около 62,5 \% прочности проводника. Учитывая результаты испытаний на растяжение, такое соединение можно считать пригодным для использования в системах, которые не подвержены действию дополнительных растягивающих нагрузок, а также для соединения электрических выводов, которые должны выдерживать нагрузки, составляющие не менее 30 \% предела прочности проводника.

Микроструктура соединения, полученного в результате термитной сварки, представлена на рис. 9. Судя по фазовым диаграммам систем $\mathrm{Cu}-\mathrm{Nb}, \mathrm{Cu}-$ $\mathrm{Sn}, \mathrm{Cu}-\mathrm{Fe}$, а также результатам микроскопического исследования и спектрального анализа, структуру термитного соединения образует медная матрица (твердый раствор олова и железа в меди), Nb фаза (точнее, твердый раствор меди в ниобии) и дисперсионная фаза Fe.

Как известно, сплав $\mathrm{Cu}-\mathrm{Nb}$ после затвердения образован из двух фаз чистых металлов. И хотя количество Fе составляет не более 1 \% от общей массы термитной смеси, однако растворимость Fе в медной матрице ограничена $0,25 \%$. Поэтому в медной матрице соединения наблюдается и фаза Fе. Такая структура получается за счет оплавления концов $\mathrm{Cu}-\mathrm{Nb}$ проводника, а также расплавления железного диска и их смешивания с жидкими продуктами термитной реакции, основу которых составляет чистая медь. Это характерная структура системы такого типа.

\section{Выводы}

Исходя из результатов данной работы, можно сделать ряд выводов. Во-первых, установлено, что путем термитной сварки при правильном подборе термитной смеси можно получить достаточно качественные соединения микрокомпозитного $\mathrm{Cu}-\mathrm{Nb}$ проводника. Механические и электриче- ские характеристики соединения, полученного методом термитной сварки, соответствуют минимальным требованиям к термитным сварным соединениям и электроконтакным соединениям. Судя по результатам исследования, подобранный состав термитной смеси обеспечивает ее хорошее возгорание, достаточно высокую температуру реакции, хорошую отделимость шлака и формирование сварного соединения микрокомпозитных $\mathrm{Cu}-\mathrm{Nb}$ проводников.

\section{Благодарность}

Авторы статьи выражают благодарность представителю компании Erico B.V. г-ну Гуннару Мустапарте за оказанную помощь и консультации в процессе подготовки термитной смеси и проведении экспериментов.

\section{Литература}

[1] Kindo, K. 2006. New pulsed magnets for 100 T, long-pulse and diffraction measurements, Journal of Physics 51: 522-528. https://doi.org/10.1088/1742-6596/51/1/118

[2] Spahn, E.; Loffler, M.; Balevičius, S. 2011. European laboratories for Pulsed power research, Journal of Korean Physical Society 59(6): 3594-3598.

[3] Herlach, F.; Miura, N. 2003. High magnetic fields. Science and technology. Vol. 1. Magnet technology and experimental techniques. London: Imperial College Press. 336 p. https://doi.org/10.1142/4764-vol1

[4] Tesanovic, Z. 2013. High magnetic field science and its application in the US: current status and future directions. Washington D.C.: National academies press. 10 p.

[5] Višniakov, N.; Novickij, J.; Ščekaturovienè, D.; Petrauskas, A. 2011. Quality analysis of welded and soldered joints of $\mathrm{Cu}-\mathrm{Nb}$ microcomposite wires, Materials Science 17(1): 16-19. https://doi.org/10.5755/j01.ms.17.1.242 
[6] Gluchovski, W.; Stobrawa, J.; Rdzawski, Z.; Masrzowski, K. 2014. Microstructure and properties of $\mathrm{Cu}-\mathrm{Nb}$ wire composites, Arhives of Metallurgy and Materials 59(1): 35-40.

[7] Shikov, A. K.; Pantsyrnyi, V.; Vorobeva, A.; Sudev, S.; Khlebova, N.; Silajev, A.; Belyakov, N. 2002. Copperniobium high strength and high conductivity winding wires for pulsed magnets, Material Science and Heat Treatment 44(11): 491-495. https://doi.org/10.1023/A:1022504805662

[8] Leprince-Wang, Y.; Han, K.; Huang, Y.; Yu-Zhang, K. 2003. Microstructure of $\mathrm{Cu}-\mathrm{Nb}$ microcomposites, Material Science and Engineering A351: 214-223. https://doi.org/10.1016/S0921-5093(02)00855-9

[9] Oguro, H.; Avaji, S.; Watanabe, K.; Sugimoto, M. 2013. New fabrication process of $\mathrm{Cu}-\mathrm{Nb}$ composite for internal reinforcement of $\mathrm{Nb} 3 \mathrm{Sn}$ wires, Journal of Superconduictivity and Novel Magnetism 26: 2099-2101. https://doi.org/0.1007/s10948-012-2031-1

[10] Rdzawski, Z.; Gluchovski, W.; Stobrawa, J.; Kempinski, W.; Andrzejewski, B. 2015. Microstructure and properties of $\mathrm{Cu}-\mathrm{Nb}$ and $\mathrm{Cu}-\mathrm{Ag}$ nanofiber composites, Archives of Civil and Mechanical Engineering 15: 689-697. https://doi.org/10.1016/j.acme.2014.12.002

[11] Gluchovski, W.; Stobrawa, J.; Rdzawski, Z.; Masrzowski, K. 2011. Mikrostructurtal charakterization of high strength high conductivity $\mathrm{Cu}-\mathrm{Nb}$ microcomposite wires, Journal of Achievements in Materials and Manufacturing Engineering 46(1): 40-49.

[12] Blumber, L.; Hasizume, H.; Ito, S.; Minervini, J.; Yanagi, N. 2010. Status of high temperature superconductuing magnet development. RSFC/JA-10-45 report. 3 p.

[13] Ciazynski, D.; Duchateau, J.; Decool, P.; Libeyre, P.; Turck, B. 2001. Large superconductors and joints for fusion magnets: from conceptual design to testing at full scale, Nuclear Fusion IAEA 41(2): 223-226. https://doi.org/10.1088/0029-5515/41/2/309

[14] Laszlo Takacs. Self-sustaining reactions induced by ball milling. Progress in Materials Science. Vol. 47, Elsevier.2002, p. 335-414.

Mikalauskas G. ${ }^{1}$, Visniakov $N .{ }^{1}$, Kvasnitsky V. V. ${ }^{2}$, Strelenko N. M. ${ }^{2}$

${ }^{1}$ Vilnius Gediminas Technical University. Vilnius, Lithuania

${ }^{2}$ National Technical University of Ukraine «Igor Sikorsky Kyiv Polytechnic Institute ». Kiev, Ukraine

\section{MICROCOMPOSITE CU-NB CONDUCTORS AND THE POSSIBILITIES OF THEIR INDIRECTABLE CONNECTION}

Installations of strong magnetic fields are widely used in various fields of scientific research and industry. The most important component of all magnetic systems is an inductor (solenoid). To create a strong magnetic field, it is no longer enough just to improve the design of solenoids. Traditional electrical materials and conductors are unable to withstand enormous mechanical and thermal loads, so they are replaced by completely new promising materials, which include microcomposite $\mathrm{Cu}-\mathrm{Nb}$ conductors. These microcomposite materials are distinguished by a unique structure, high mechanical characteristics and good electrical conductivity. One of the most important unsolved problems remains the creation of reliable permanent connections and the search for new technologies for their manufacture, since the majority of such magnetic installations should remain inseparable after the start of their operation. At present, to connect conductors, usually only detachable threaded or soldered joints are used, which do not differ in practice with high reliability. This paper presents an overview of: features of the structure and characteristics of $\mathrm{Cu}$ - $\mathrm{Nb}$ conductors used in installations for strong magnetic fields; the specifics of the production of these composite conductors; technological problems at their connection by welding; the results of experimental studies of the properties of welded joints, which were performed by thermite welding. [dx.doi.org/10.29010/084.6]

Keywords: $\mathrm{Cu}-\mathrm{Nb}$ conductors; electrocontact connections; microcomposite materials; welded joints.

\section{References}

[1] Kindo, K. 2006. New pulsed magnets for 100 T, long-pulse and diffraction measurements, Journal of Physics 51: $522-528$. https://doi.org/10.1088/1742-6596/51/1/118

[2] Spahn, E.; Loffler, M.; Balevičius, S. 2011. European laboratories for Pulsed power research, Journal of Korean Physical Society 59(6): 3594-3598. 
[3] Herlach, F.; Miura, N. 2003. High magnetic fields. Science and technology. Vol. 1. Magnet technology and experimental techniques. London: Imperial College Press. 336 p. https://doi.org/10.1142/4764-vol1

[4] Tesanovic, Z. 2013. High magnetic field science and its application in the US: current status and future directions. Washington D.C.: National academies press. $10 \mathrm{p}$.

[5] Višniakov, N.; Novickij, J.; Ščekaturovienè, D.; Petrauskas, A. 2011. Quality analysis of welded and soldered joints of Cu-Nb microcomposite wires, Materials Science 17(1): 16-19. https://doi.org/10.5755/j01.ms.17.1.242

[6] Gluchovski, W.; Stobrawa, J.; Rdzawski, Z.; Masrzowski, K. 2014. Microstructure and properties of Cu-Nb wire composites, Arhives of Metallurgy and Materials 59(1): 35-40.

[7] Shikov, A. K.; Pantsyrnyi, V.; Vorobeva, A.; Sudev, S.; Khlebova, N.; Silajev, A.; Belyakov, N. 2002. Copperniobium high strength and high conductivity winding wires for pulsed magnets, Material Science and Heat Treatment 44(11): 491-495. https://doi.org/10.1023/A:1022504805662

[8] Leprince-Wang, Y.; Han, K.; Huang, Y.; Yu-Zhang, K. 2003. Microstructure of Cu-Nb microcomposites, Material Science and Engineering A351: 214-223. https://doi.org/10.1016/S0921-5093(02)00855-9

[9] Oguro, H.; Avaji, S.; Watanabe, K.; Sugimoto, M. 2013. New fabrication process of Cu-Nb composite for internal reinforcement of Nb3Sn wires, Journal of Superconduictivity and Novel Magnetism 26: 2099-2101. https://doi.org/0.1007/ s10948-012-2031-1

[10] Rdzawski, Z.; Gluchovski, W.; Stobrawa, J.; Kempinski, W.; Andrzejewski, B. 2015. Microstructure and properties of Cu-Nb and $\mathrm{Cu}-\mathrm{Ag}$ nanofiber composites, Archives of Civil and Mechanical Engineering 15: 689-697. https://doi.org/ 10.1016/j.acme.2014.12.002

[11] Gluchovski, W.; Stobrawa, J.; Rdzawski, Z.; Masrzowski, K. 2011. Mikrostructurtal charakterization of high strength high conductivity $\mathrm{Cu}-\mathrm{Nb}$ microcomposite wires, Journal of Achievements in Materials and Manufacturing Engineering 46(1): 40-49.

[12] Blumber, L.; Hasizume, H.; Ito, S.; Minervini, J.; Yanagi, N. 2010. Status of high temperature superconductuing magnet development. RSFC/JA-10-45 report. 3 p.

[13] Ciazynski, D.; Duchateau, J.; Decool, P.; Libeyre, P.; Turck, B. 2001. Large superconductors and joints for fusion magnets: from conceptual design to testing at full scale, Nuclear Fusion IAEA 41(2): 223-226. https://doi.org/10.1088/0029-5515/41/2/309

[14] Laszlo Takacs. Self-sustaining reactions induced by ball milling. Progress in Materials Science. Vol. 47, Elsevier.2002, p. 335-414. 